Particle Accelerators, Vol. 62, pp. [563-588]/43-68 Reprints available directly from the publisher Photocopying permitted by license only
(C) 1998 OPA (Overseas Publishers Association) N.V.

Published by license under the Gordon and Breach Science

Publishers imprint. Printed in India.

\title{
THE TESLA PROJECT
}

\author{
BJÖRN H. WIIK* \\ Deutsches Elektronen-Synchrotron and University of Hamburg \\ (II. Institute of Physics), D-22607, Hamburg, Germany
}

(Received in final form 15 January 1998)

\begin{abstract}
Studies towards a next generation $\mathrm{e}^{+} \mathrm{e}^{-}$linear collider based on superconducting linac technology (TESLA) are performed by an international collaboration co-ordinated at DESY. This technology combines a high efficiency of acceleration with optimum beam quality and thus offers a high performance in terms of the achievable luminosity. In addition, an X-ray Free Electron Laser and a continuous electron beam source for Nuclear Physics are foreseen to be integrated into the linear collider project. The lecture describes the layout of the TESLA facility and the progress on R\&D towards high-performance superconducting accelerating structures.
\end{abstract}

Keywords: Superconductivity; Radiofrequency; Cavities; Collider

\section{INTRODUCTION}

During the past four years an international collaboration centred at DESY has worked on the layout of TESLA, ${ }^{1}$ an $\mathrm{e}^{+} \mathrm{e}^{-}$collider based on low frequency superconducting rf cavities. The TESLA collaboration has also carried out a large $R \& D$ programme aimed at improving the performance and reducing the cost of superconducting rf cavities.

The TESLA $\mathrm{e}^{+} \mathrm{e}^{-}$collider facility will also be a source of coherent $\mathrm{X}$-rays with tunable wavelengths in the Ångström region. Combined with the HERA electron ring it can furthermore provide an intense, high duty cycle polarized electron beam at energies up to $25 \mathrm{GeV}$.

* Tel.: 49-40-8998-2407/2408. Fax: 49-40-8994-4304.

E-mail: BJOERN.WIIK@DESY.DE. 
In this lecture I, will first discuss the layout and the performance of the TESLA facility, then review the status of the R\&D programme, and end with a few remarks on the realization of the facility. The focus will be on TESLA as an $\mathrm{e}^{+} \mathrm{e}^{-}$collider.

\section{THE TESLA FACILITY}

A schematic layout of the TESLA facility is shown in Figure 1.

A train of low emittance electron or positron bunches is extracted from a source system, compressed longitudinally, and injected into two opposing linear accelerators. The bunches are carefully aligned along the machine axis as defined by the quadrupole magnets and the rf cavities and accelerated to $250 \mathrm{GeV}$. After acceleration the beam halo is removed by a set of precision collimators, and the bunches are transported and focussed down to a few hundred $\mathrm{nm}$ in the horizontal direction and at the order of $10 \mathrm{~nm}$ in the vertical plane and collided head on. The large bunch spacing makes it possible to use a bunch-tobunch feedback system to ensure that opposing bunches collide head-on at the interaction point (IP). The spent beams are extracted from the interaction point and used to produce the next batch of positrons and electrons.

Interleaved with the bunches for particle physics, bunches of electrons originating from a low emittance rf photocathode gun are longitudinally compressed and accelerated to energies between 10 and $50 \mathrm{GeV}$. They are then extracted from the linac, transported to the surface and passed through long, high precision undulators. The low emittance electron bunches traversing the undulators will yield a very bright, very short burst of transversely coherent light with tunable wavelengths in the Ångström region. A total of 11315 bunches spaced by $93 \mathrm{~ns}$ are accelerated during each rf pulse. Using a transverse mode cavity to deflect the beam it is possible to feed several undulators during the same rf pulse. The electron beam energy may also be varied from bunch train to bunch train and consequently such a facility can serve a large number of users without interfering with the particle physics programme.

To realize an X-ray laser facility in the Ångström region requires multi-GeV electron beams of unprecedented quality. Furthermore, 


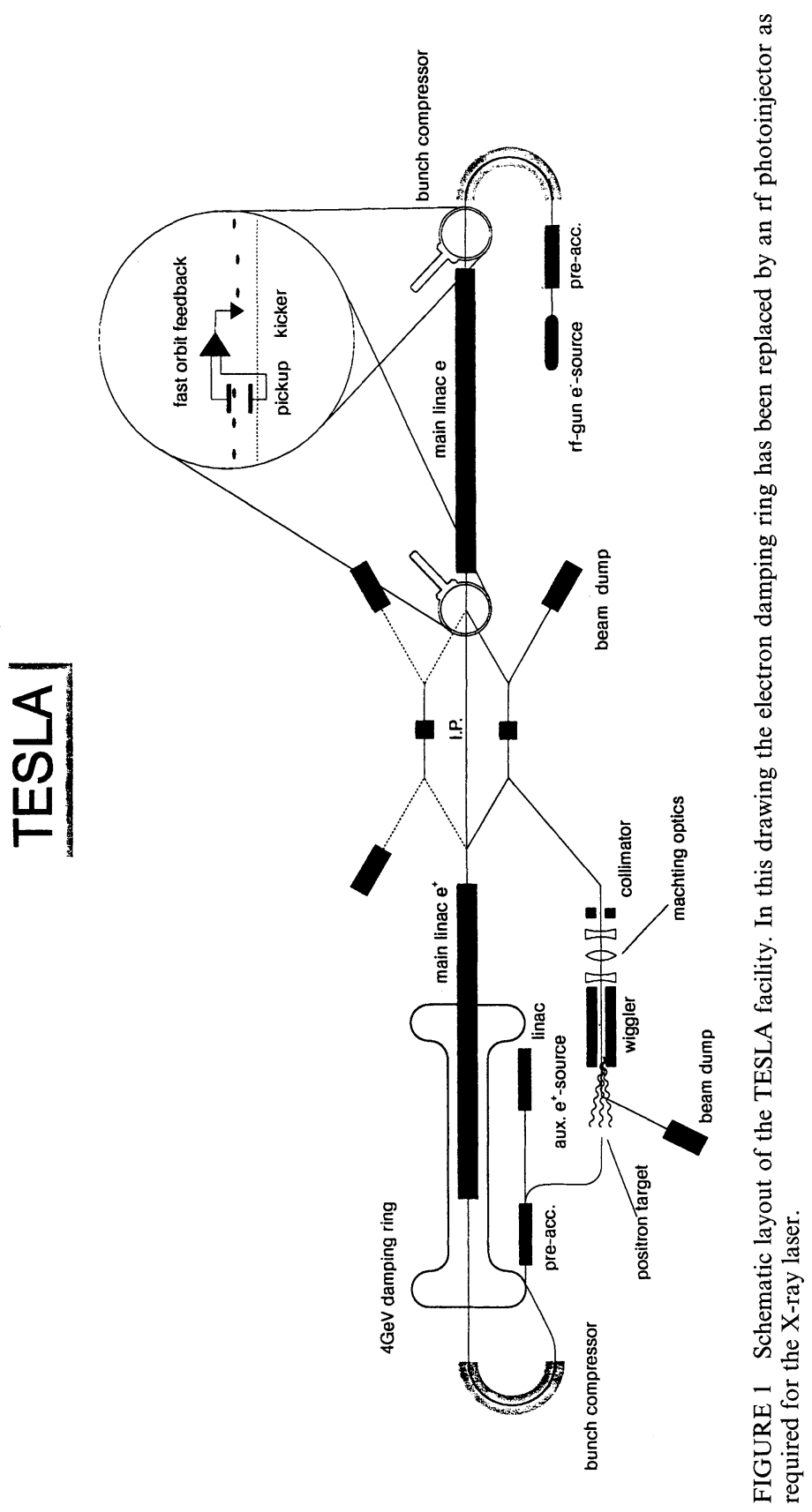


a large and well trained staff is needed to construct, commission and operate the facility and a large site is required to produce, handle and use the intense photon beams. Thus combining the laser facility with the $\mathrm{e}^{+} \mathrm{e}^{-}$collider facility will lead to a substantial reduction both in construction and operation cost.

TESLA, combined with the HERA electron ring, can also be used to produce a high intensity, high duty cycle electron beam in the energy range from 15 to $25 \mathrm{GeV}$. In this scheme ${ }^{1,2}$ additional electron bunches are accelerated in the first part of the TESLA positron linac and injected into the HERA electron ring at the chosen energy. The electrons are extracted over ms yielding a $30 \mu \mathrm{A}$ electron beam with a duty cycle of $85 \%$ and an energy resolution of order $10^{-3}$. An additional advantage of HERA is that electrons may be polarized.

The combined duty cycle is of order $3 \%$. The facility can thus serve all users without interference provided that the $\mathrm{rf}$ system and the cryogenic system feeding the first part of the linear accelerator are upgraded compared to what is needed for the $\mathrm{e}^{+} \mathrm{e}^{-}$collider alone.

To study ep collisions in the multi- $\mathrm{TeV}$ range electrons from a high energy, high duty cycle linear accelerator must be collided with the protons in a storage ring. Since TESLA will be using standing wave cavities, electrons and positrons can be accelerated in the opposite directions in the same linac and thus electrons accelerated in the first part of the positron linac can be made to collide with the protons in HERA yielding a c.m. energy of roughly $1.3 \mathrm{TeV}$. This ep-option ${ }^{3}$ will not be discussed further.

\section{WHY SUPERCONDUCTING CAVITIES?}

The advantages of basing the main linac on superconducting low frequency cavities follow directly from the luminosity formula:

$$
L=5.82 \cdot 10^{20} \cdot \frac{\eta \cdot P_{\mathrm{w}}}{E_{\mathrm{c} . \mathrm{m} .}} \cdot\left(\frac{\delta_{\mathrm{E}}}{\xi_{y} \varepsilon_{y}^{\mathrm{N}}}\right)^{1 / 2} \cdot H_{\mathrm{D}}\left(\mathrm{cm}^{-2} \mathrm{~s}^{-1}\right) .
$$

In this formula the $\mathrm{e}^{+} \mathrm{e}^{-}$c.m. energy is denoted by $E_{\text {c.m. }}, P_{\mathrm{w}}$ is the mains supply power and $\eta$ is the overall efficiency for converting mains supply power into beam power, $\varepsilon_{y}^{\mathrm{N}}$ is the normalized vertical beam emittance at 
the source and $\xi_{y}$ is the emittance dilution between source and interaction point, and $\delta_{\mathrm{E}}$ is the fractional energy loss due to bremsstrahlung. $H_{\mathrm{D}}$, the disruption enhancement factor caused by the pinch effect at collision is of order 1.5-2. This luminosity formula is based on the natural assumption that the vertical beta-function at the interaction point is equal to the bunch length, i.e. $\beta_{y}^{*}=\sigma_{z}$.

In the expression for the luminosity the c.m. energy $E_{\text {c.m. }}$ and the fractional energy loss are both determined by the needs of the experiment. Thus for a given mains supply power $P_{\mathrm{w}}$ the luminosity formula contains only two free parameters: $\eta$ and $\xi_{y} \cdot \varepsilon_{y}^{\mathrm{N}}$, the normalized vertical beam emittance at the interaction point. Both parameters favour the use of low frequency, high $Q$-value superconducting cavities.

The $Q$-value of a superconducting rf cavity is roughly $10^{6}$ times larger than the $Q$-value of a normal conducting cavity yielding a corresponding reduction in $\mathrm{rf}$ wall losses. A high accelerating gradient in a superconducting cavity can thus be established and maintained at low rf input power resulting in a large value of $\eta$. Furthermore, the high $Q$-value makes it possible to choose a low rf frequency and a large bunch spacing which minimizes wakefield effects.

The advantage of a low frequency superconducting accelerator can be demonstrated ${ }^{4}$ by comparing the alignment tolerances of TESLA with those of an X-band accelerator.

When the beam traverses the accelerating structure it will induce longitudinal and transverse wakefields which act back on the bunch itself or on trailing bunches. The strengths of the transverse and the longitudinal wakefield are proportional to the cube and the square of the rf frequency, respectively, hence the magnitude of the unavoidable emittance dilution between damping ring and interaction point depends strongly on the choice of the rf frequency.

The single bunch transverse emittance dilution caused by the short range wakefield can be written as:

$$
\frac{\Delta \varepsilon}{\varepsilon}=F \cdot \bar{\beta} \cdot \mathrm{d} y_{\mathrm{c}}^{2},
$$

where $\bar{\beta}$ denotes the average $\beta$-function in the linac, $\mathrm{d} y_{\mathrm{c}}^{2}$ is the rms offset of the cavities with respect to the linac axis and $F$ is the dilution factor. The values of $F$ as evaluated ${ }^{5}$ for the various proposed linear colliders TESLA $(1.3 \mathrm{GHz})$, SLC $(3.0 \mathrm{GHz})$, SBLC $(3.0 \mathrm{GHz})$, NLC 


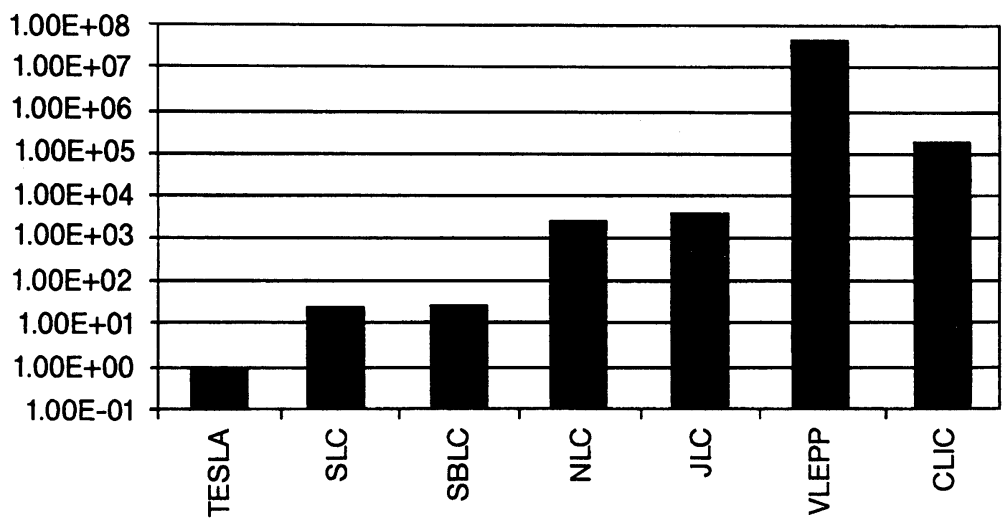

FIGURE 2 The single bunch wakefield strength function $F$ evaluated for different linear collider designs.

(11.4 GHz), JLC (11.4 GHz), VLEPP (14.6 GHz) and CLIC $(30.0 \mathrm{GHz})$ are shown in Figure 2. The required rms alignment tolerances are $500 \mu \mathrm{m}$ for TESLA and of order $10 \mu \mathrm{m}$ for the X-band. In the case of the $\mathrm{X}$-band design this requires strong focussing and $\mathrm{BNS}^{6}$ damping.

The effects of long range wakefields can be neglected in the case of TESLA. In the case of the higher frequency designs they can be controlled by the use of damped and detuned structures.

The strong focussing and the correlated momentum spread along the bunch required by the $\mathrm{X}$-band design leads to strong dispersion and chromatic effects which dilute the emittance. This in turn leads to tight tolerances ${ }^{4}$ of the order of $1-3 \mu \mathrm{m}$ for the positioning accuracy of the quadrupoles and the beam position monitors in the case of the X-band compared to $100 \mu \mathrm{m}$ for TESLA.

Clearly the base line TESLA design is rather conservative and it seems possible to increase ${ }^{7}$ the luminosity by at least one order of magnitude as discussed below.

\section{THE TESLA FACILITY}

\subsection{The $\mathrm{e}^{+} \mathrm{e}^{-}$Collider}

The schematic layout of the TESLA collider ${ }^{1}$ is shown in Figure 1 and its baseline parameters are listed in Table I. 
TABLE I Baseline TESLA parameters

\begin{tabular}{lccc}
\hline c.m. energy $(\mathrm{GeV})$ & 500 & 800 & 1600 \\
Total length $(\mathrm{km})$ & 33 & 33 & 62 \\
Gradient $(\mathrm{MV} / \mathrm{m})$ & 25 & 40 & 40 \\
$n_{\mathrm{b}}$ per rf pulse & 1130 & 2260 & 2260 \\
Bunch spacing $(\mathrm{ns})$ & 708 & 283 & 283 \\
Repetition rate $(\mathrm{Hz})$ & 5 & 3 & 3 \\
$\mathrm{~N}_{\mathrm{e}} /$ bunch $\left(10^{10}\right)$ & 3.63 & 1.82 & 1.82 \\
$\varepsilon_{x} / \varepsilon_{y}\left(10^{-6} \mathrm{~m} \cdot \mathrm{rad}\right)$ & $14 / 0.25$ & $12 / 0.025$ & $12 / 0.025$ \\
$\beta_{x}^{*} / \beta_{y}^{*}(\mathrm{~mm})$ & $25 / 0.7$ & $25 / 0.5$ & $25 / 0.5$ \\
$\sigma_{x}^{*} / \sigma_{y}^{*}(\mathrm{~nm})$ & $845 / 19$ & $618 / 4.0$ & $436 / 2.8$ \\
$\sigma_{z}(\mathrm{~mm})$ & 0.7 & 0.5 & 0.5 \\
$\delta_{\mathrm{E}}(\%)$ & 2.5 & 2.2 & 6.7 \\
Number of klystrons & 616 & 1232 & 2464 \\
$P_{\mathrm{b}}(2$ beams $)(\mathrm{MW})$ & 16.3 & 15.6 & 31.2 \\
$P_{\mathrm{AC}}(2$ linacs $)(\mathrm{MW})$ & 99 & 115 & 230 \\
Luminosity $\left(10^{33} \mathrm{~cm}^{-2} \mathrm{~s}^{-1}\right)$ & 6 & 11 & 20 \\
\hline
\end{tabular}

The basic building block of the linear accelerator is a $12.2 \mathrm{~m}$ long cryogenic module. The module consists of eight $1.035 \mathrm{~m}$ long nine-cell cavities installed in a common cryostat. The module may also include a beam position monitor and steering and focussing magnets. The $1.3 \mathrm{GHz}$ cavity made from high RRR niobium sheets is mounted in a helium container and is equipped with an $\mathrm{rf}$ power coupler, two higher order mode couplers and a mechanical tuning system driven by a step motor.

Each linear accelerator is made of 1232 cryogenic modules. Including the $2.2 \mathrm{~km}$ long beam delivery sections, the total site length is of order $33 \mathrm{~km}$.

The $\mathrm{rf}$ power to the cavities is provided by 616 klystrons, each of $10 \mathrm{MW}$ peak power feeding $4 \times 8$ cavities. The nominal peak $\mathrm{rf}$ power is $216 \mathrm{~kW} / \mathrm{m}$ leaving a $20 \%$ reserve to compensate for phase changes due to mechanical deformation of the cavities caused by the Lorentz force.

A total of 1130 bunches spaced by $708 \mathrm{~ns}$ are accelerated within the $1.33 \mathrm{~ms}$ long rf pulse and this is repeated at a rate of $5 \mathrm{~Hz}$. The charge per bunch is $3.63 \cdot 10^{10}$ electrons (positrons) leading to $4 \cdot 10^{13}$ electrons (positrons) per rf pulse. It seems rather difficult to produce the required number of positrons for the bunch trains using a conventional source. It is thus proposed to pass the spent beam through a wiggler yielding a bright burst of high energy photons. After the beam depart 
from the wiggler, they are deflected into a beam dump, whereas the photon beam strikes a target producing a train of positron bunches which are preaccelerated by a conventional L-band linac followed by a superconducting linac to $200 \mathrm{MeV}$ and $3.2 \mathrm{GeV}$, respectively.

The trains of positron (electron) bunches are stored in two dog-boneshaped damping rings with a circumference of roughly $17 \mathrm{~km}$. The bunch spacing is roughly $50 \mathrm{~ns}$. Each damping ring consists of two $8 \mathrm{~km}$ long straight sections placed entirely in the linear accelerator tunnel connected with two loops at the ends. Damping times of some $20 \mathrm{~ms}$ are obtained using four $100 \mathrm{~m}$ long wigglers. Detailed calculations show that the required emittances can be achieved. Indeed, it seems feasible to produce beams with a vertical emittance more than an order of magnitude lower than presently required by either reducing the circumference of the ring or by raising its energy and by using beam based alignment techniques to centre the beam.

The bunches are extracted from the damping ring, compressed longitudinally to $0.7 \mathrm{~mm}$ in a single stage, and injected into the main linac with the proper bunch spacing.

In order to reach a high luminosity the beam must be accelerated through the linear accelerator with a minimum of emittance dilution to the final energy of $250 \mathrm{GeV}$. Several effects contribute to the emittance dilution, single and multibunch wakefield effects, dispersion effects due to the energy spread of the beam and vibrations.

Extensive tracking calculations were done assuming the components to be aligned to the following rms tolerances: cavities $500 \mu \mathrm{m}$, quadrupoles and beam position monitors to $100 \mu \mathrm{m}$. The emittance growth is below $15 \%$ using a simple beam steering algorithm. With advanced beam based alignment methods this number can be reduced to a few percent.

Due to the large bunch spacing and cavity-to-cavity detuning and higher order mode damping the limit for beam breakup is quite high. For an rms multibunch energy spread of order $5 \cdot 10^{-4}$ the multibunch emittance growth is negligibly small.

An optimal trajectory through the linac is found through a beam based alignment. Effects due to the slow ground motion are cancelled out using a slow feedback system. The orbit must be corrected 
only once a day and every 2 years the optimal trajectory must be reestablished.

To correct for the fast motion one takes advantage of the fact that the bunches are spaced far apart. Beam monitors measure the position of the first few bunches and the centroid positions of the remaining bunches in the train are then centred using fast deflecting correctors as indicated in Figure 1. This results in an acceptable quadrupole vibration tolerance of order $1 \mu \mathrm{m}$ which is well above the measured amplitudes of order $0.1-0.2 \mu \mathrm{m}$.

After traversing the linear accelerator the beam is collimated, corrected and passed on to the final focussing system. In the final focus system the beam will be demagnified by a factor of 100 . Demagnification factors of more than 300 have already been achieved at the final focus beam test facility ${ }^{8}$ at SLAC.

For the X-ray laser a low emittance bunch train is produced in a special source system consisting of an $\mathrm{rf}$ photoinjector, a longitudinal bunch compression system and a $3 \mathrm{GeV}$ linear accelerator. This system delivers a bunch train consisting of 11315 bunches spaced by $93 \mathrm{~ns}$ and each with a charge of $1 \mathrm{nC}$. At the entrance to the main linac the rms bunchlength is $23 \mu \mathrm{m}$ which corresponds to a peak current of $5 \mathrm{kA}$ and the invariant transverse emittance is $1 \pi \mathrm{mm}$ mrad. Downstream along the linac two independent beams are extracted with energies tunable in the energy range between 10 and $25 \mathrm{GeV}$ and between 25 and $50 \mathrm{GeV}$, respectively, transported over a distance of roughly $10 \mathrm{~km}$ and brought to the surface. At the surface the beam is distributed among three independent beam lines by means of a fast transverse mode cavity feeding a total of 4 SASE $^{9}$ undulators and 6 spontaneous undulator beam lines as shown in Figure 3. Each photon beam line can feed 5 experimental stations - i.e. a total of 50 experiments can in principle be carried out simultaneously.

By installing a small linac section in front of each section it is possible to fine tune the energy $( \pm 2 \%)$. It is also possible to vary intensity, polarization etc. within the bunch train. Typical photon beam parameters are listed in Table II.

The temporal intensity of a single ESRF pulse is compared schematically in Figure 4 with the intensity delivered by a single TESLA SASE pulse. 


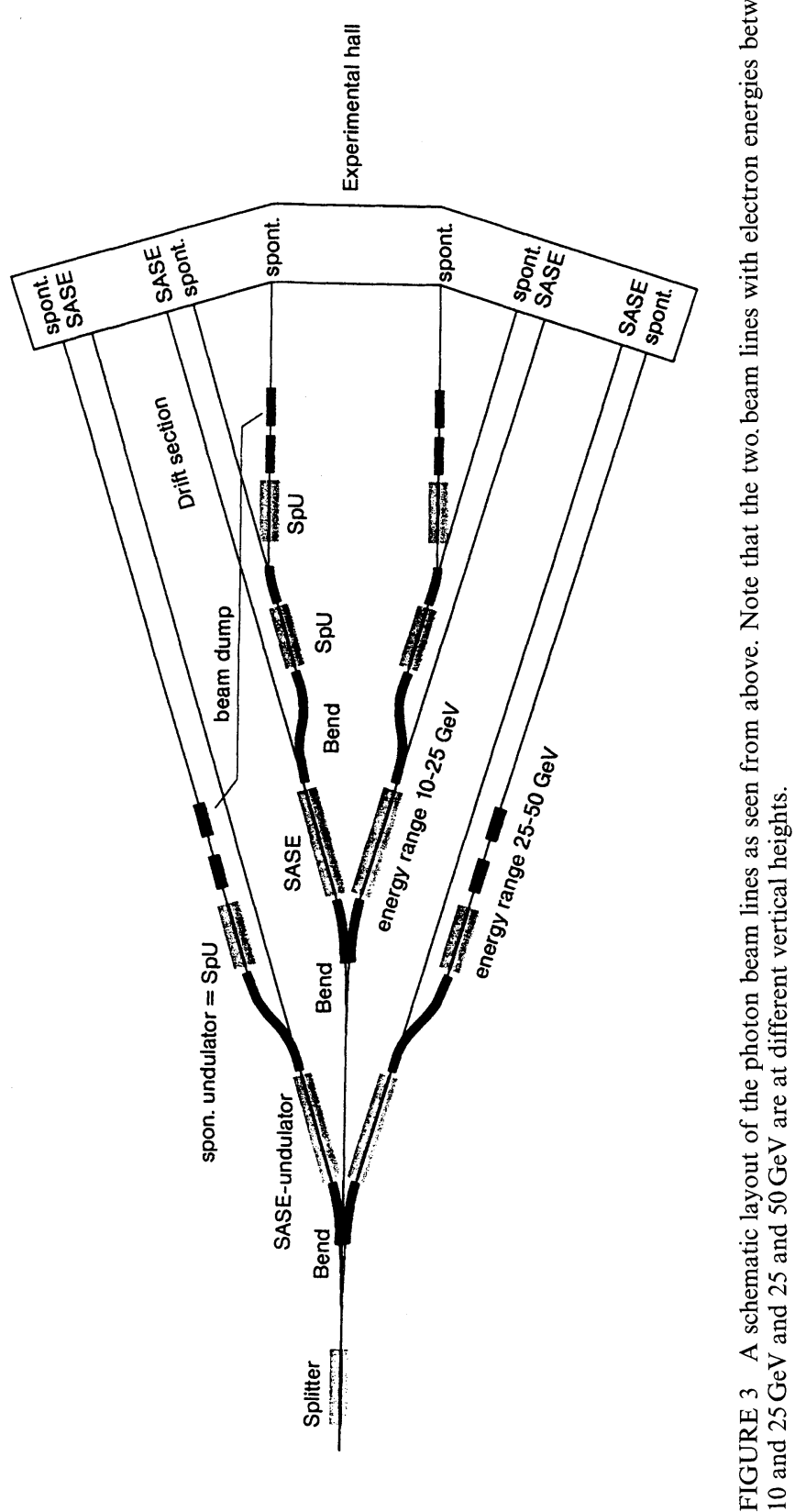


TABLE II Photon beam parameters

Typical undulator saturation length (m)

Peak spectral brilliance*

Average spectral brilliance*

$50-100 \mathrm{~m}$

$10^{34}$

Photon energy range (keV)

$10^{26}$

Photon beam power (GW)

$0.1-12 \mathrm{keV}$

Number of photons/bunch

$60-280$

rms bunch width (fs)

$10^{12}-10^{14}$

Transverse coherence 200

Photon beam divergence

1

$1 \mu \mathrm{rad}$

Photon beam size at exit chamber

$20 \mu \mathrm{m}$

${ }^{*}$ The brilliance is quoted in number of photons $\cdot\left[\mathrm{s} \cdot \mathrm{mrad}^{2} \cdot \mathrm{mm}^{2} \cdot(0.1 \% \text { bandwidth })\right]^{-1}$

$$
\lambda=1 \AA
$$

$10^{13}$ photons

FEL

100 fs

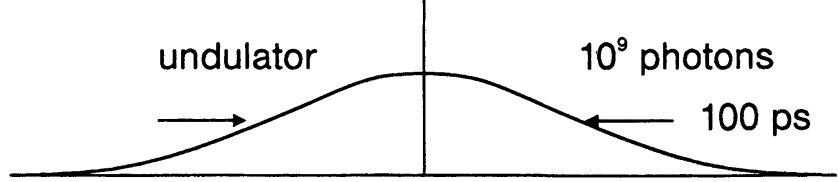

FIGURE 4 Comparison between an ESRF undulator pulse and a TESLA SASE single pass pulse (in units of photons $\cdot\left[\mathrm{s} \cdot \mathrm{mrad}^{2} \cdot \mathrm{mm}^{2} \cdot(0.1 \% \text { bandwidth) }]^{-1}\right)$. 
Peak and average brilliance for three single pass FELs and for three possible undulators are plotted in Figure 5(a) and (b) and compared to the performance of some third generation sources.

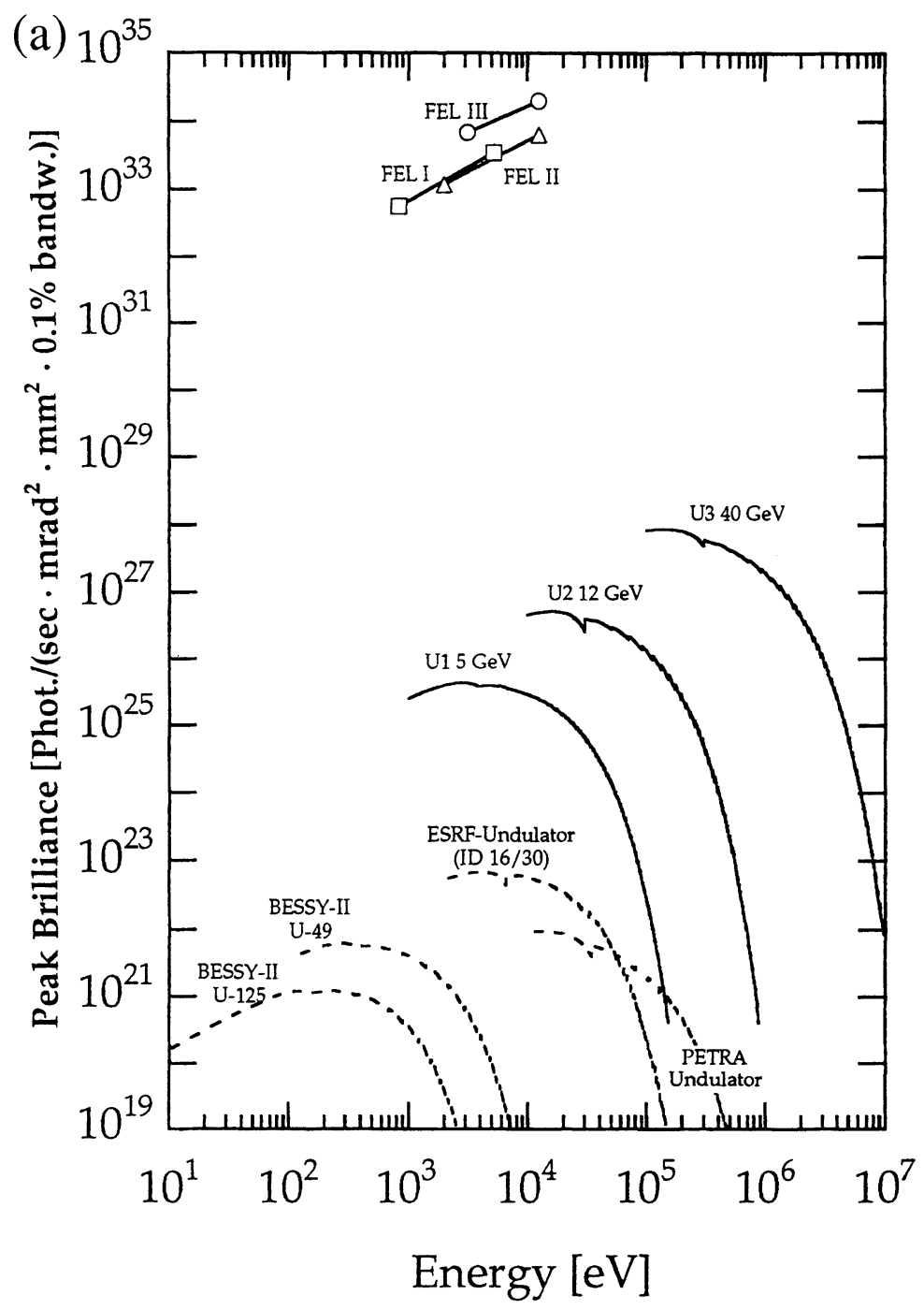

FIGURE 5(a) 


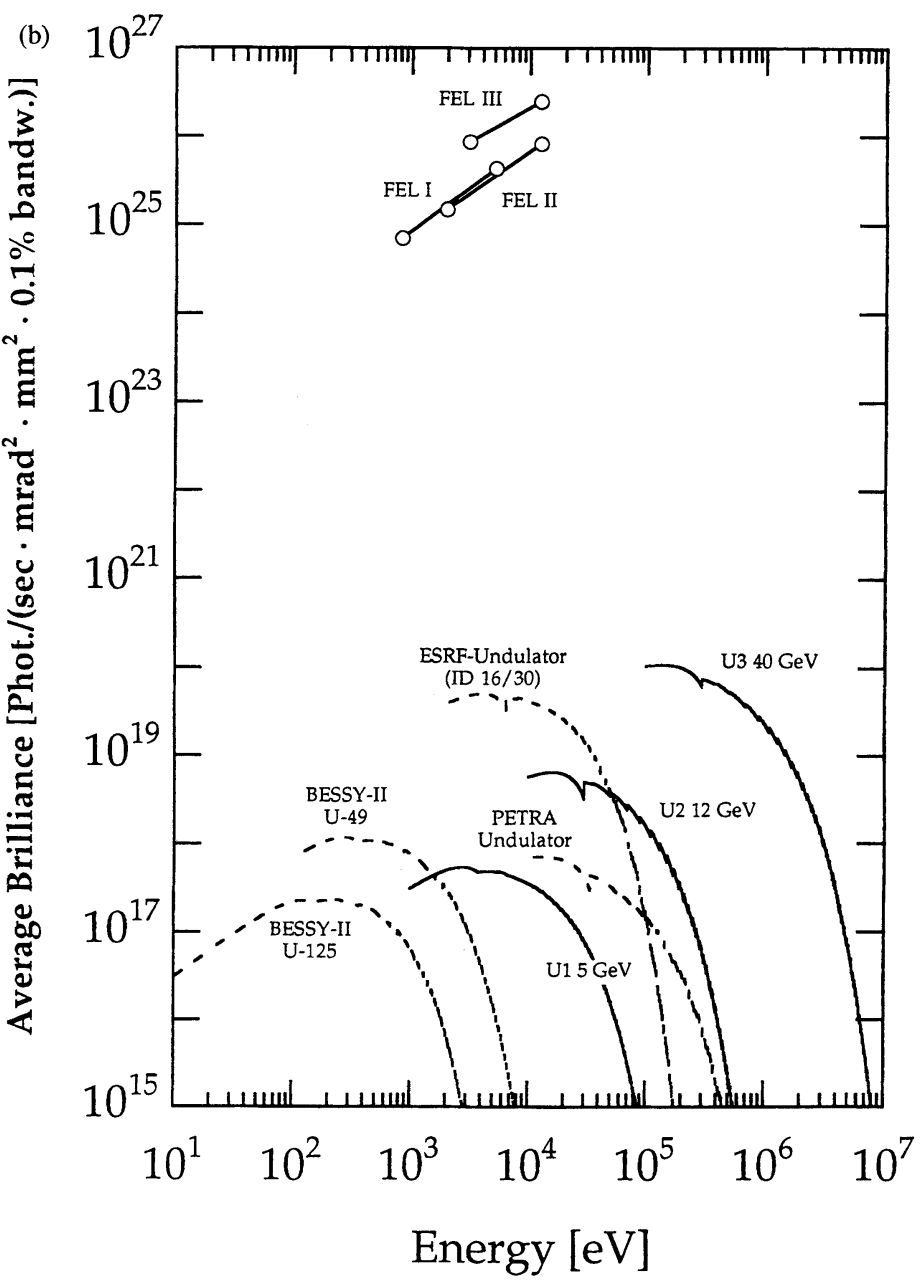

FIGURE 5(b)

FIGURE 5 Peak brilliance (a) and average brilliance (b) plotted versus wavelength for the three FELs proposed and for three possible undulators. For comparison the peak brilliance produced by conventional storage ring undulators are also shown.

\subsection{Energy and Luminosity}

The maximum voltage gradient of a superconducting cavity is around $55 \mathrm{MV} / \mathrm{m}$, limited by the strength of the magnetic field at the equator 
of the cavity. This is well above the gradient of $25 \mathrm{MV} / \mathrm{m}$ assumed for TESLA 500. However, in the past accelerating gradients reached in multicell cavities have been limited to values around $5-10 \mathrm{MV} / \mathrm{m}$ by field emission from localized regions of the cavity surface, by thermal quenches induced by inclusions of foreign material in the $\mathrm{Nb}$ material or by the quality of cavity weld joints. The TESLA R\&D programme has shown that gradients of $25 \mathrm{MV} / \mathrm{m}$ can be reached in solid $\mathrm{Nb}$ cavities based on present production techniques and on the quality of $\mathrm{Nb}$ sheets now available. Based on the steady progress in the performance of superconducting cavities it seems reasonable to assume that in the next decade cavities with accelerating gradients of order $40 \mathrm{MV} / \mathrm{m}$ will be at hand. Gradients close to $40 \mathrm{MV} / \mathrm{m}$ have already been reached ${ }^{10}$ in single cell cavities.

Replacing $25 \mathrm{MV} / \mathrm{m}$ cavities with $40 \mathrm{MV} / \mathrm{m}$ cavities yields a c.m. energy of $800 \mathrm{GeV}$. A preliminary set of parameters for an $800 \mathrm{GeV}$ $\mathrm{e}^{+} \mathrm{e}^{-}$collider is listed in Table $\mathrm{I}$.

Higher energies can also be reached by increasing the length of the accelerator. A very preliminary list of parameters for a $1.6 \mathrm{TeV}$ collider some $64 \mathrm{~km}$ long can also be found in Table I. The proposed DESY site can accomodate a $75 \mathrm{~km}$ long linear accelerator.

It seems possible to increase ${ }^{11}$ the TESLA filling factor from its present value of $67 \%$ to a value of $80 \%$ by reducing the space between adjacent cavities in a module and by reducing the space allocated to cryogenic equipment. Keeping the length of the linac constant, this would imply an increase in the c.m. energy from $500 \mathrm{GeV}$ to $580 \mathrm{GeV}$, or from $800 \mathrm{GeV}$ (resp. $1600 \mathrm{GeV}$ ) to $920 \mathrm{GeV}$ (resp. $1940 \mathrm{GeV}$ ). Conversely nominal energies may now be reached at a gradient of 20.6 and $33 \mathrm{MV} / \mathrm{m}$, respectively.

It has also been recently proposed ${ }^{12}$ to use the superconducting linear collider as a part of a muon collider. In this case multi-TeV $\mu^{+} \mu^{-}$ collisions seem feasible. However, numerous technical problems inherent to the construction of a muon collider are still unsolved. A fundamental problem is the intense neutrino flux resulting from the decay of the muons which represent a potential health hazard and may well limit the luminosity at high energies.

The physics potential of an $\mathrm{e}^{+} \mathrm{e}^{-}$collider depends strongly on the integrated luminosity. The quoted peak luminosity of $6 \cdot 10^{33} \mathrm{~cm}^{-2} \mathrm{~s}^{-1}$ corresponding to a yearly integrated luminosity of $50 \mathrm{fb}^{-1}$, is based 
TABLE III Possible scenarios for the luminosity upgrade ${ }^{7}$

\begin{tabular}{lcccc}
\hline General parameters & Ref. design & Stage I & Stage II & Stage III \\
\hline No. of bunches per pulse & 1130 & 1410 & 2820 & 4028 \\
Pulse length $(\mu \mathrm{s})$ & 800 & 950 & 950 & 950 \\
Bunch spacing $\Delta t_{\mathrm{b}}(\mathrm{ns})$ & 708 & 674 & 337 & 236 \\
Bunch charge $N\left(10^{10}\right)$ & 3.63 & 4.0 & 2.0 & 1.4 \\
Pulse current $(\mathrm{mA})$ & 8.2 & 9.5 & 9.5 & 9.5 \\
Av. beam power $(\mathrm{MW})$ & 8.3 & 11.3 & 11.3 & 11.3 \\
Emittance at IP $\varepsilon_{x, y}\left(10^{-6} \mathrm{~m}\right)$ & $14,0.25$ & $14,0.25$ & $10,0.03$ & $8,0.0075$ \\
$\beta_{x, y}$ at IP $(\mathrm{mm})$ & $25,0.7$ & $25,0.7$ & $15,0.4$ & $12.7,0.3$ \\
Spot size at IP $\sigma_{x, y}(\mathrm{~nm})$ & 845,19 & 845,19 & 558,5 & $459,2.1$ \\
Bunch length at IP $\sigma_{z}(\mathrm{~mm})$ & 0.7 & 0.7 & 0.4 & 0.3 \\
Bremsstrahlung $\delta_{\mathrm{B}}(\%)$ & 25 & 3.0 & 3.0 & 3.0 \\
Vert. disruption $D_{y}$ & 18 & 20 & 33 & 50 \\
Luminosity $\left(10^{34} \mathrm{~cm}^{-2} \mathrm{~s}^{-1}\right)$ & 0.6 & 0.9 & 2.6 & 5.2 \\
\hline
\end{tabular}

on conservative assumptions as discussed above. Because of the high efficiency for converting rf power into beam power and the low wakefields inherent to low frequency superconducting cavities, it seems feasible ${ }^{7}$ to increase the luminosity of TESLA to values of order $5 \cdot 10^{34} \mathrm{~cm}^{-2} \mathrm{~s}^{-1}$ for a constant mains supply power of $100 \mathrm{MW}$ and with $\delta_{\mathrm{B}} \approx 3 \%$.

The higher luminosity is reached by increasing the efficiency $\eta$ by a factor of 1.5 to a value of $23 \%$ and by lowering the beam emittance by a factor of 50 . The former is the result of reducing the gradient to $20.6 \mathrm{MV} / \mathrm{m}$ while the decrease in beam emittance requires a redesign of the damping rings. The new, very preliminary parameters are listed in Table III.

\section{STATUS OF THE R\&D PROGRAMME}

\subsection{The R\&D Facility}

The aim of the R\&D programme ${ }^{13}$ is to establish the technical base needed to construct and operate a high energy $\mathrm{e}^{+} \mathrm{e}^{-}$linear collider made of superconducting cavities and to demonstrate that this collider can be realized in a cost effective manner and that its performance meets the design goals. We also aim to demonstrate that the SASE principle can be used to produce coherent light with $\mathrm{nm}$ wavelengths. 
TABLE IV Members of the TTF-collaboration

\begin{tabular}{ll}
\hline China & IHEP Beijing, Tsinghua University Beijing \\
Finland & SEFT \\
France & CEA/DSM Saclay, IN2P3 Orsay \\
Germany & RWTH Aachen, MBI Berlin, DESY, GH Wuppertal, \\
& Univ. of Frankfurt, GKSS Geesthacht, FZ Karlsruhe, \\
& TH Darmstadt, TU Berlin, TU Dresden \\
Italy & INFN Frascati, INFN Milano, INFN Roma II \\
Poland & Acad. of Science Warsaw, Univ. of Warsaw, Univ. of Cracow, \\
& Univ. of Mining \& Metallurgy Cracow, Atomic Energy Agency Warsaw, \\
& Soltan Institute Otwock-Swierk \\
Russia & JINR Dubna, IHEP Protvino, INP Novosibirsk \\
USA & Cornell University Ithaca NY, Fermilab Batavia IL, UCLA, \\
& Los Angeles, CA
\end{tabular}

The institutions participating in this programme are listed in Table IV.

\subsection{Layout and Performance of the Cavities}

A picture of a nine-cell resonator made from solid $\mathrm{Nb}$ sheets is shown in Figure 6 and the main cavity parameters are listed in Table V.

The complete cavities are produced by industry starting from high purity niobium sheets $2.5 \mathrm{~mm}$ thick. The raw niobium material is purified using electron beam melting and the remaining contamination is of the order of a few ppm for $\mathrm{O}_{2}$ and $\mathrm{N}_{2}$ and roughly $100 \mathrm{ppm}$ in the case of Ta. After the mechanical treatment, the damaged surface layer is removed by etching and the sheets are heat treated to reach the required residual resistivity ratio of 250 or more and to produce a uniform grain size.

So far, 24 cavities which have been produced by European firms, have been processed and tested at temperatures down to $1.8 \mathrm{~K}$ at the DESY facility. A detailed discussion can be found in contributions to this conference; here I summarize the main results only.

Some $40 \%$ of all cavities measured so far reach gradients in excess of $20 \mathrm{MV} / \mathrm{m}$. For these cavities the $Q$-value versus the accelerating gradient are plotted in Figure 7. The average gradient and average $Q$-value of $23 \mathrm{MV} / \mathrm{m}$ and $1.7 \cdot 10^{10}$, respectively in the continuous wave mode exceed the design goal of $15 \mathrm{MV} / \mathrm{m}$ and $3 \cdot 10^{9}$ and are approaching the performance needed to construct TESLA. 


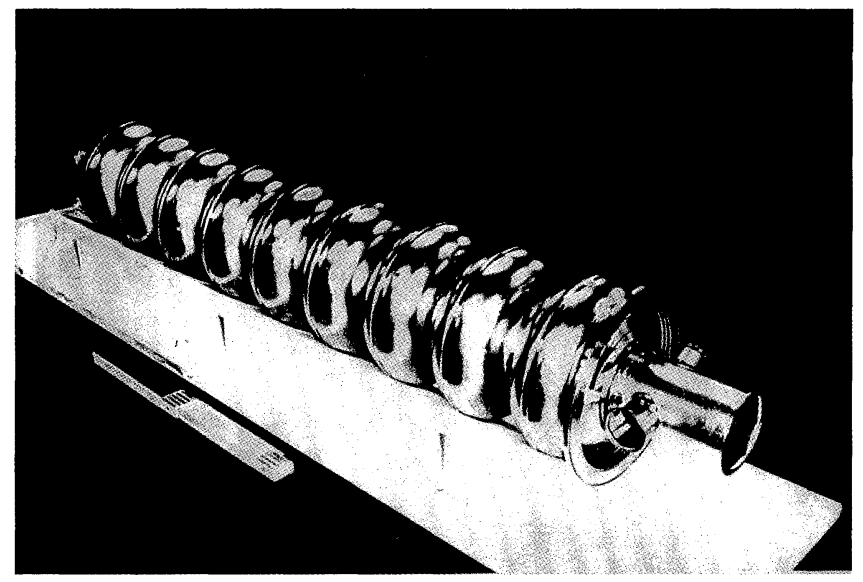

FIGURE 6 Superconducting nine-cell TESLA cavity made from solid $\mathrm{Nb}$ sheets.

TABLE V Cavity design parameters

\begin{tabular}{lc}
\hline Frequency & $1.3 \mathrm{GHz}$ \\
Number of cells/cavity & 9 \\
Operating temperature & $1.8 \mathrm{~K}$ \\
$Q_{0}$ at $15 \mathrm{MV} / \mathrm{m}$ & $\geq 3 \cdot 10^{9}$ \\
$R / Q$ & $973 \Omega / \mathrm{m}$ \\
Accelerating field & $15 \mathrm{MV} / \mathrm{m}$ \\
$E_{\text {peak }} / E_{\text {acc }}$ & 2.0 \\
$B_{\text {peak }} / E_{\text {acc }}$ & $4.2 \mathrm{mT} / \mathrm{MV} / \mathrm{m}$ \\
Cavity length & $1.03 \mathrm{~m}$ \\
\hline
\end{tabular}

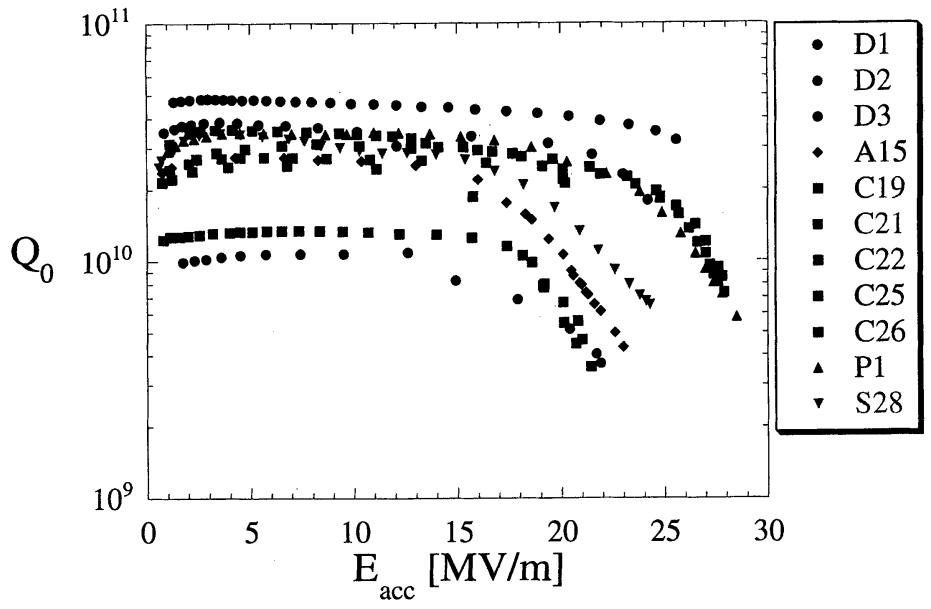

FIGURE 7 Quality factor versus gradient for all cavities with a limiting gradient of $20 \mathrm{MV} / \mathrm{m}$ or more. Some $40 \%$ of the cavities tested meet this criterium. The performance of A15, C21, C25, C26, S28, P1 is limited by the amplifier. 


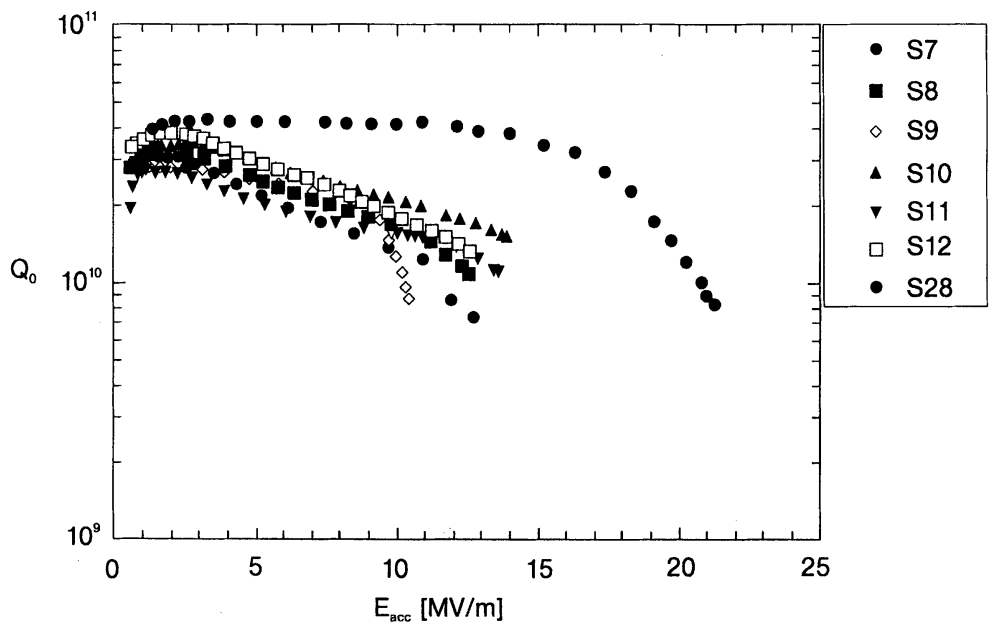

FIGURE 8 Quality factor versus gradient for cavities produced by a single vendor. Cavity S28 was produced using an improved welding procedure.

The performance of the remaining cavities seems to be limited mainly by:

- problems occurring during manufacturing,

- impurity inclusions in the material.

In Figure 8 the observed $Q$-value is plotted versus the accelerating gradient for cavities marked $\mathrm{S}$ which were produced by a single vendor.

The $Q$-value for six of these cavities decreases rapidly with increasing gradient leading to a quench at gradients between 10 and $15 \mathrm{MV} / \mathrm{m}$. It was demonstrated by temperature mapping that the quenches occur at the weld joints and it was found that they were caused by an inadequate welding procedure. The performance of S28 which is the first cavity produced by the same vendor using an improved welding procedure is also plotted in Figure 8. Note the strikingly different $Q$-behaviour of this cavity as a function of gradient. The maximum observed gradient of $24.5 \mathrm{MV} / \mathrm{m}$ is limited by the available $\mathrm{rf}$ input power.

The performance of the remaining cavities is in general limited by a quench in a single cell. The location of the quench was determined by temperature mapping and it was found that it was in general caused by impurity inclusions in the material. Only 6 cells out of the 54 
cells in the $6 \mathrm{D}$ cavities listed above fail to reach a gradient of $20 \mathrm{MV} / \mathrm{m}$. Thus 6 out of the $108 \mathrm{Nb}$ sheets used in the production of the $\mathrm{D}$ cavities had presumably inclusions which limited the cavity performance.

The 26 cavities now under production will all be fabricated using pretested $\mathrm{Nb}$ sheets and improved quality control.

\subsection{The TESLA Test Accelerator}

The layout of the TESLA test accelerator ${ }^{13}$ is shown in Figure 9 and its parameters are listed in Table VI.

Injector I, made of a thermionic gun providing a $250 \mathrm{keV} 8 \mathrm{~mA}$ beam, a $217 \mathrm{MHz}$ buncher cavity and a nine-cell standard TESLA superconducting cavity as a capture cavity, has been installed and commissioned.

Recently the first cryomodule containing 8 cavities was installed and cooled down to $1.8 \mathrm{~K}$. The static heat load at $1.8 \mathrm{~K}$ is of order $0.5 \mathrm{~W} / \mathrm{m}$. Unfortunately one of the stepping motors used to tune the cavities at cryogenic temperature was blocked during the first series of experiments. The remaining 7 cavities have been tuned and powered in parallel by a common rf source.

A beam with the design current of $8 \mathrm{~mA}$ was accelerated to an energy of $125 \mathrm{MeV}$ corresponding to an average peak gradient of $16.7 \mathrm{MV} / \mathrm{m}$. The observed quench limit corresponds to an average peak gradient of $19.4 \mathrm{MV} / \mathrm{m}$. The flat top of the rf pulse was $100 \mu \mathrm{s}$ long and the beam was accelerated and transported to the beam dump some $40 \mathrm{~m}$ downstream of the module exit with $100 \%$ transmission. The rms energy spread was $3 \cdot 10^{-3}$. So far no deleterious effects due to ground vibrations were observed. The performance of the first module thus meets or exceeds the design goals.

\section{TABLE VI TTF linac design parameters}

\begin{tabular}{lc}
\hline Linac energy (MeV) & 390 \\
RF frequency $(\mathrm{GHz})$ & 1.3 \\
Accelerating gradient $(\mathrm{MV} / \mathrm{m})$ & 15 \\
Unloaded quality factor & $3 \cdot 10^{9}$ \\
Number of cryomodules & 3 \\
$\Delta \mathrm{E} / \mathrm{E}$ single bunch rms & $10^{-3}$ \\
$\Delta \mathrm{E} / \mathrm{E}$ bunch to bunch rms & $2 \cdot 10^{-3}$ \\
Bunch length & $1 \mathrm{~mm}$ \\
Beam macropulse length & $800 \mu \mathrm{s}$ \\
\hline
\end{tabular}



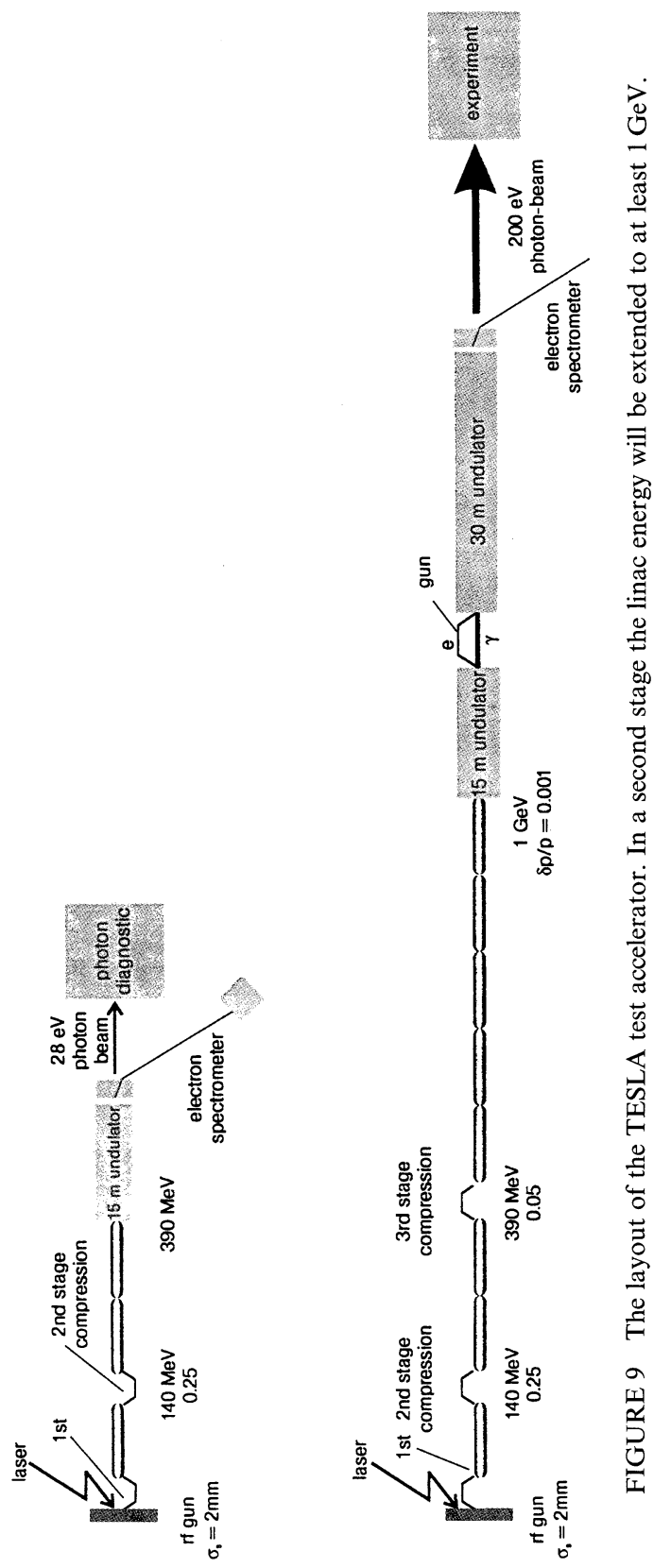
The remaining two modules will be installed in early autumn of 1998. By that time the rf photoinjector capable of providing a beam with the TESLA design parameters will become available. This facility will then serve as an integrated system test and will also provide a wealth of data on the performance of individual subsystems. Besides operational issues the experiments will also address crucial beam dynamics issues.

With this facility it will also be possible to extend the measurements of the single pass SASE (Self Amplified Spontaneous Emission) process ${ }^{9}$ down to wavelengths of order $40 \mathrm{~nm}$.

For this purpose the facility will be augmented by the following components:

- A low emittance, high bunch rate rf photoinjector.

- Two stages of bunch compressors yielding a $0.25 \mathrm{~mm}$ long bunch at the entrance of the undulator.

- A $15 \mathrm{~m}$ long undulator with an undulator wavelength of $27.3 \mathrm{~mm}$ and a magnetic fieldstrength of $0.497 \mathrm{~T}$.

- Instrumentation.

The construction and commissioning of these components is well advanced and the first SASE experiments are scheduled for the spring of 1999.

After the completion of the test programme at TTF I it is planned to raise the energy to at least $1 \mathrm{GeV}$ by installing additional modules. These modules will be installed in a $5.2 \mathrm{~m}$ diameter tunnel as foreseen for TESLA. This linac serves a dual purpose:

- Provide an integrated system test of new and improved components and systems.

- Driver of a SASE FEL with wavelengths down to $6 \mathrm{~nm}$ and below.

The second stage of the TTF will be based on the experience from TTF I and incorporate novel features:

- The electron beam welding of the cups is a costly and delicate step in the cavity production process. Indeed, faulty welds seem to be the main reason why cavities do not reach fields above $20 \mathrm{MV} / \mathrm{m}$. Two methods - hydroforming and spinning - are presently being 
explored. Palmieri and his coworkers at Legnaro have successfully produced $^{14}$ two single-cell cavities, one four-cell cavity and one fivecell cavity by spinning. The first one-cell cavity has been tested and has reached a gradient of $24.5 \mathrm{MV} / \mathrm{m}$ at a $Q$-value of $3 \cdot 10^{9}$. This cavity was made of $\mathrm{Nb}$ sheet with a RRR of 300 . A Nb cell has also been successfully produced by hydroforming. It is planned to equip a part of the modules with seamless cavities.

- It seems possible ${ }^{11}$ to feed three to four seven-cell cavities with a single power coupler. Tests of this concept are now underway and if successful a few of the modules will be based on this concept.

- The cavities will be excited by a high efficiency $(\geq 70 \%)$ multibeam klystron now under development in industry. The first test data are encouraging and we expect the first klystron of this type to be delivered in 1998.

- This klystron will be powered by a novel type of modulator with the energy stored in a superconducting solenoid. A first prototype modulator based on this principle is now being developed at Forschungszentrum Karlsruhe and should be available in 1999.

- Based on experience with the TESLA Test Facility several other components are being slightly modified. We thus expect that this second iteration will result in an accelerator which in layout and performance will be very close to the final TESLA design.

After a series of experiments which will provide information on TESLA in its collider mode, this linac will be used as a driver of a $30 \mathrm{~m}$ long undulator providing coherent light with the first harmonic at $6 \mathrm{~nm}$. The schematic layout of the facility is shown in Figure 9.

The expected peak brilliance of this facility is plotted in Figure 10 versus photon energy. Note that the brilliance can be raised by two orders of magnitude using a seed laser. As a seed laser we plan to pass the electron beam through a $15 \mathrm{~m}$ long undulator and pass the light output directly to the main undulator while delaying the electron bunches by the appropriate amount. The high brilliance, coupled with the short pulse length and the $100 \%$ coherence will open up new experimental possibilities in many areas. After a runningin period an extensive experimental programme is planned. Such a programme is mandatory in order to be able to utilize the TESLA X-ray facility. 


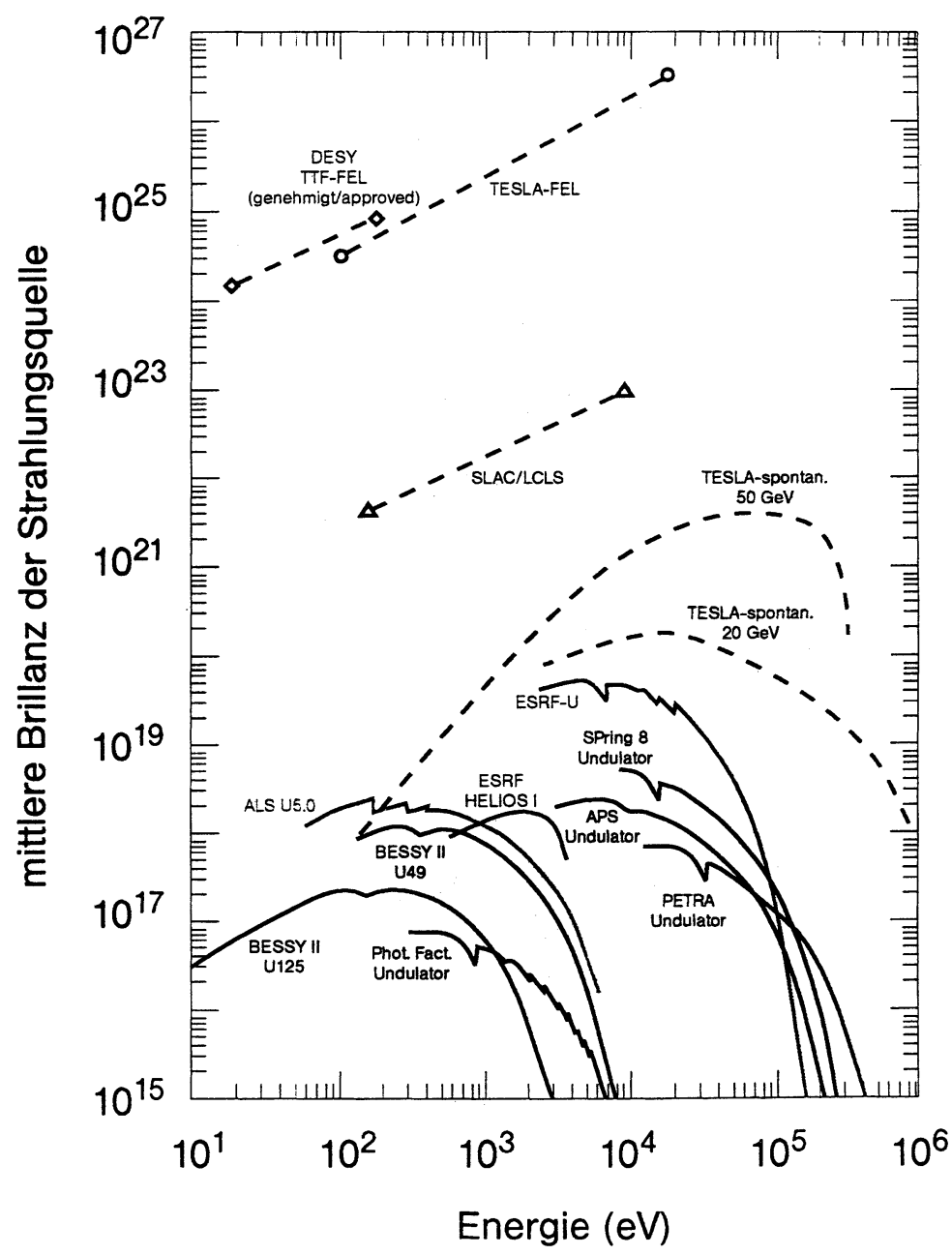

FIGURE 10 Average brilliance of the TTF FEL in comparison with the performance of some third generation light sources. The average brilliance of the proposed SLAC FEL is also shown.

\section{OUTLOOK}

A Conceptual Design Report covering the accelerator and its scientific potential is now available. Members from more than 50 Institutes in 12 countries have collaborated in writing this report. 
This report will be the basis for a detailed engineering design of the TESLA facility and it also affords a detailed comparison with other approaches.

Two potential sites - one at FERMILAB and one at DESY - have been explored. Both sites are well suited from a geological point of view and have the additional advantage of offering a large well trained staff and substantial infrastructure which can be integrated into the project.

The layout of the accelerator at the DESY site is shown in Figure 11. The $5.2 \mathrm{~m}$ diameter tunnel starts on the DESY site and runs north

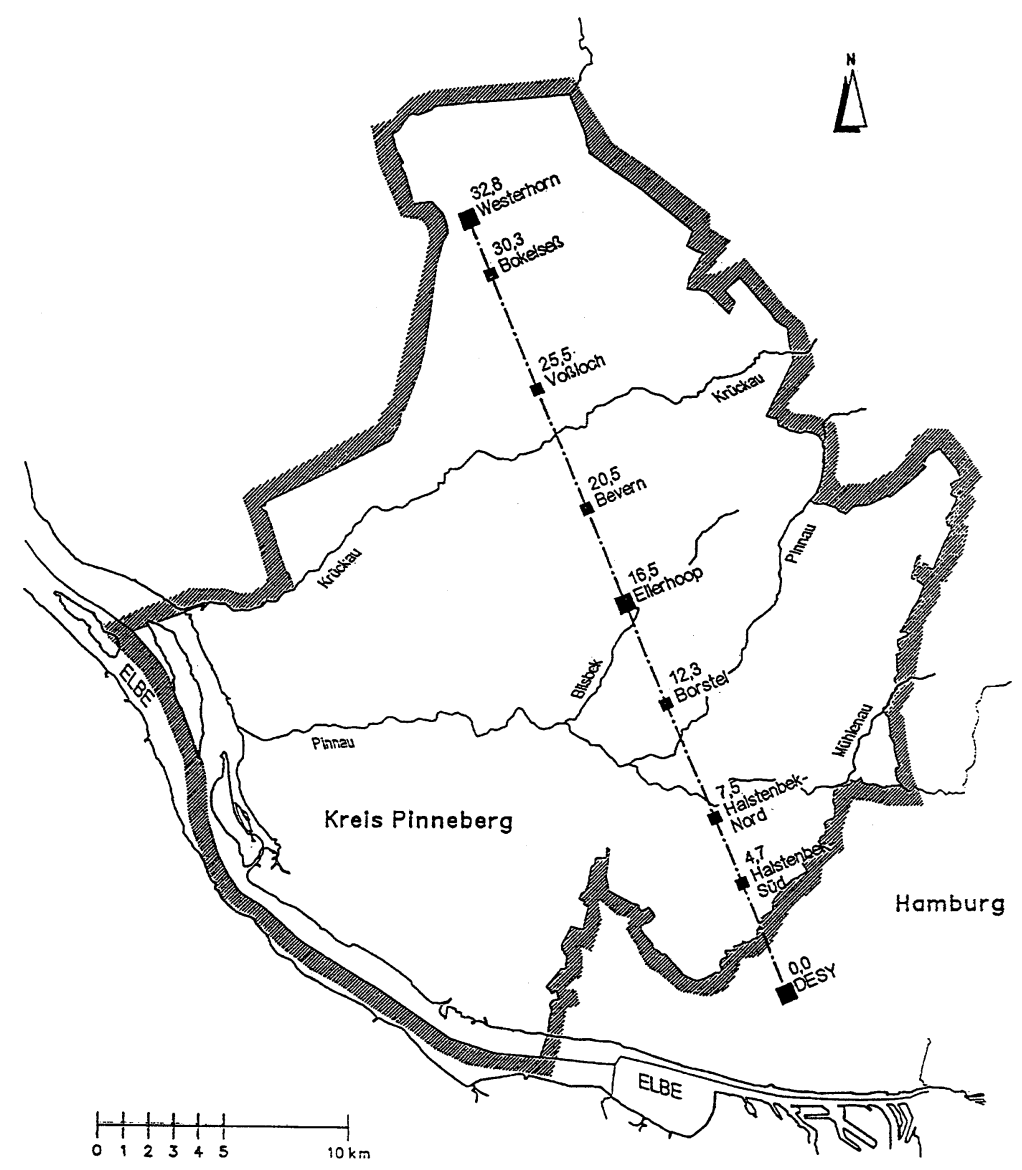

FIGURE 11 Potential site for the linear collider. 
along straight section west of HERA for a total length of $33 \mathrm{~km}$. The collision hall and the facilities for the synchrotron radiation users are on a common campus situated $16.5 \mathrm{~km}$ to HERA hall west. A surface building every $6 \mathrm{~km}$ is needed to house the refrigeration plant and the modulators. The location of these buildings is also indicated.

For the main part the tunnel follows the earth's curvature at an average depth of $15 \mathrm{~m}$. It passes through sand below the water table and will be built using the same tunnel boring technology as used for the construction of HERA.

A joint committee set up by the governments in SchleswigHolstein and Hamburg has defined the legal framework for the construction of this facility. Work on an environmental impact report has started.

The final proposal including cost and time schedule will be based on the successful operation of the test facility and on industrial studies of the main components. We expect that this proposal will be completed by 2001. We have estimated that the facility as proposed can be constructed in 7 years.

\section{References}

[1] R. Brinkmann, G. Materlik, J. Rossbach and A. Wagner (Eds.), Conceptual design of a $500 \mathrm{GeV} \mathrm{e}^{+} \mathrm{e}^{-}$linear collider with integrated X-ray laser facility, DESY Report 1997-048; ECFA Report 1997-182 (This report also includes a complete set of references.); G. Ingelman and L. Jönsson (Eds.), Future electron accelerators and free electron lasers - prospects and opportunities in natural sciences, Nuclear Instruments and Methods RD 9, 348 (1997) 1-108.

[2] R. Brinkmann, DESY TESLA 95-14.

[3] M. Tigner, B.H. Wiik and F. Willeke, Proc. 1991 IEEE, Part. Acc. Conf., San Francisco, May 1991, Vol. 5 (1992), p. 2916.

[4] A. Mosnier, Proc. XVIII International Symposium on Lepton-Photon Interactions, Hamburg (1997), in press.

[5] R. Brinkmann, DESY Report M-95-10, (1995).

[6] V.E. Balakin, A. Novokhatsky and V. Smirnov, Proc. of the 12th Int. Conf. on High Energy Acc. (1983), p. 119.

[7] R. Brinkmann, DESY TESLA 97-13.

[8] D. Burke, Final focus test beam design report, SLAC Report 376 (1990), Proc. 4th European Part. Acc. Conf., London, 1994, Vol. I, p. 23.

[9] A.M. Kondratenko and E.E. Saldin, Part. Acc., 10 (1980) 207; R. Bonifacio, C. Pellegrini and L.M. Narducci, Optics Communications, 50 (1989) 373.

[10] P. Kneisel, R.W. Röth and H.-G. Kurschner, Proceedings of the 7th Workshop on RF-Superconductivity, Saclay, 1995, p. 449; M. Ono et al., Proc. of the 21st Linear Accelerator Meeting, NUP-A-96-16, 36, Tokyo (1996). 
[11] J. Sekutowicz, Private communications.

[12] D. Neuffer, H. Edwards and D. Finley, Proc. 1997 IEEE Part. Acc. Conf., Vancouver (1997), in press.

[13] D.A. Edwards (Ed.), A proposal to construct and test prototype superconducting RF structures for linear colliders, DESY Internal Report, April 1992, TESLA Test Facility Linac, TESLA 95-01 (1995).

[14] See V. Palmieri, Contribution to this conference. 